\title{
Konstitutioneller Minimalismus: die EU-Sozialpolitik in den Vertragsreformen von Nizza bis Lissabon
}

\author{
Hans-Wolfgang Platzer*
}

Die wissenschaftlichen und politischen Urteile über die vertragliche Verankerung und weitere Ausgestaltung der EU-Sozialpolitik in den konstitutionellen Reformbemühungen seit dem Vertrag von Nizza bewegen sich - bezogen auf die Ergebnisse des Reformvertrages von Lissabon - zwischen einer Position, die die graduellen Fortschritte insbesondere im Bereich der allgemeinen sozialpolitischen Zielbestimmungen betont, einer Position, die einen Stillstand insbesondere in den ,materiellen' Vertragsbestimmungen sieht, und einer Position, nach der der Reformvertrag de facto einen Rückschritt markiert. Dies insofern, als eine auf längere Sicht letzte Chance zu einer nachhaltigen Stärkung der arbeits- und sozialpolitischen Kompetenzausstattung der Union vertan sei und damit die Dominanz einer wettbewerbsgetriebenen ,Marktintegration` zementiert werde.

Diese unterschiedlichen Bewertungen der sozialpolitischen Reformergebnisse sind unter den gesellschaftlichen Kräften Europas (Gewerkschaften, NGOs, Netzwerke wie Attac, etc.) vielfach auch ausschlaggebend für deren Akzeptanz oder Ablehnung des Vertragswerkes insgesamt.

Die hier unternommene Analyse setzt sich mit der Frage auseinander, ob und inwieweit in den Verhandlungsprozessen zur EU-Sozialpolitik seit Nizza, deren Ergebnisse als konstitutioneller Minimalismus qualifiziert werden, zugleich die vertragspolitischen Grenzen einer weiteren Ausgestaltung der sozialen Dimension in der EU-27 insgesamt sichtbar werden. Zur empirischen Unterfütterung und historischen Einordnung dieses Prozesses werden zunächst die Entwicklung der Vertragsgrundlagen und die bisherige materielle Ausgestaltung der EU-Sozialpolitik sowie die Grundzüge des gegenwärtigen sozialpolitischen Regierens (in) der Europäischen Union skizziert.

Jegliche Analyse dieses Themenkomplexes steht gegenwärtig, Ende des Jahres 2008, unter einem doppelten Vorbehalt. Denn nach dem irischen ,Nein` zum Reformvertrag ist dessen Zukunft ebenso ungewiss, wie es nach der jüngsten dramatischen Weltfinanzmarktkrise derzeit kaum absehbar ist, inwieweit diese globalen Erschütterungen den bis dato dominierenden neoliberalen wirtschaftspolitischen Diskurs in Europa und das sozial-ökonomische Regieren (in) der Europäischen Union - auch jenseits aller konstitutionellen Reformen - beeinflussen und verändern werden.

Das heißt, es ist angesichts des ,Schattens der Zukunft', der derzeit konstitutionell, realwirtschaftlich und politisch über Europa liegt und sich weiter auszubreiten scheint, nicht auszuschließen, dass sich die Auseinandersetzung mit der Sozialpolitik im Vertragswerk von Lissabon letztlich als ,historische Fußnote" erweisen könnte.

Gleichwohl mag der Versuch lohnen, Verlauf und Ergebnisse der Vertragsverhandlungen im Bereich der Sozialpolitik zu analysieren, weil sich darin grundlegendere Probleme der institutionellen Gesamtarchitektur der Europäischen Union spiegeln und eine komplexe Gemengelage aus ordnungs- und integrationspolitischen Interessendivergenzen und Zielkonflikten sichtbar wird. Das heißt, die Ausgestaltung des Vertragskapitels zur Sozialpolitik der Union kann nicht isoliert, sondern nur in Relation zu und im Zusammenhang mit jenen EU-

Prof. Dr. Hans-Wolfgang Platzer, Jean Monnet Chair of European Integration, Hochschule Fulda. 
Kompetenzfeldern betrachtet werden - Wettbewerbs- und Steuerpolitik, wirtschafts- und währungspolitische Vertragsbestimmungen, etc. - die für die Sozialpolitikentwicklung und Wohlfahrtsproduktion auf mitgliedstaatlicher Ebene bedeutsame Rahmenbedingungen setzen.

Denn gerade die Ausgestaltung dieser, angrenzenden ' beziehungsweise mit der Sozialpolitik verflochtenen Politikfelder entscheidet darüber - zum Teil stärker als die genuinen sozialpolitischen Vertragsbestimmungen selbst -, ob sich die Europäische Union als Ganzes in Richtung einer wettbewerbsstaatlichen Ordnung oder einer sozialstaatlichen Ordnung bewegt.

Unter diesem breiteren Horizont einer bereits nach Maastricht begonnenen Debatte über die ,konstitutionelle ' Einbettung der Währungsunion durch den weiteren Ausbau supranationaler wirtschaftspolitischer Steuerungs- und sozialpolitischer Regulierungskapazitäten der Union, setzt sich die Analyse vor allem mit Problemstellungen auseinander, die Grundsatzfragen der weiteren Entwicklung der Europäischen Union berühren:

Stößt die Europäische Union im vertragspolitisch-konstitutionellen Bereich an die Grenzen einer Reform ihres sozial-ökonomischen Kompetenzgefüges?

Lassen sich die Verhandlungsverläufe seit dem Konvent und die Ergebnisse von Lissabon dahingehend deuten, dass sich die erweiterte Union in einer Kompetenztransferfalle ${ }^{1}$ befindet?

Sind Formen einer flexiblen, abgestuften Integration oder extrakonstitutionelle Problemlösungspfade Auswege aus dieser Kompetenztransferfalle und werden sie in Zukunft an Bedeutung gewinnen?

\section{Historische Vertragsentwicklungen, das Verhältnis von ,positiver ${ }^{6}$ und ,negativer Integration und der sozialpolitische acquis communautaire}

Um eine historische Grundlage für die Analyse des Post-Nizza-Prozesses zu schaffen und theoriegeleitete Maßstäbe für die Auseinandersetzung mit den sozialpolitischen Ergebnissen des Lissabon-Vertrages zu gewinnen, gilt es die Bedingungen und Ausprägungen des sozialökonomischen Regierens (in) der Europäischen Union kurz aufzufächern.

Die Unterscheidung zwischen ,negativen" (im weitesten Sinne ,marktschaffenden') und ,positiven" (im weitesten Sinne ,marktkorrigierenden' beziehungsweise gemeinschaftliche Politik konstituierenden) Regelungen transnationaler sozial-ökonomischer Handlungszusammenhänge spielt hierbei in der integrationswissenschaftlichen Debatte eine zentrale Rolle. Mit Blick auf die Probleme einer sozialpolitischen Regulierung durch die Europäische Union hat Scharpf eine weitere wichtige Unterscheidung hinzugefügt: „Die Grenze zwischen konsensfähigen und konflikthaften (Interessen-)Konstellationen [lässt] sich näherungsweise durch die Unterscheidung zwischen produkt- und mobilitätsbezogener Regelungen auf der einen und der Harmonisierung produktions- und standortgebundener Regelungen auf der anderen Seite ziehen [...] Der letztlich ausschlaggebende - und vorderhand nicht ausräumbare Grund für die Konflikthaftigkeit von produktions- und standortbezogenen Regelungen liegt in den extremen Unterschieden im ökonomischen Entwicklungsstand der Mitgliedstaaten.“2

1 Dieser Begriff lehnt sich bewusst an die „Politikverflechtungsfalle“ an, mit der Scharpf die damaligen Stagnationstendenzen und Entscheidungsblockaden im Mehrebenensystem der Europäischen Union beschrieben hat. Vgl. Fritz W. Scharpf: Die Politikverflechtungs-Falle: Europäische Integration und deutscher Föderalismus im Vergleich, in: Politische Vierteljahresschrift 4/1985, S. 323-356.

2 Fritz W. Scharpf: Politische Optionen im vollendeten Binnenmarkt, in: Markus Jachtenfuchs/Beate KohlerKoch (Hrsg.): Europäische Integration, Opladen 1996, S. 109-140, hier S. 112. 
Die Grundentscheidungen darüber, wie und in welcher Gewichtung sich die ,positive und ,negative" Integration gestaltet, werden in den jeweiligen Vertragsverhandlungen getroffen, das heißt im Primärrecht der Verträge durch die Kompetenzorganisation und die Entscheidungsregeln fixiert. Integrationsgeschichtlich mutet es paradox an, dass keine der großen politischen Auseinandersetzungen seit den 1950er Jahren, in denen sich das politische Selbstbewusstsein Europas geformt hat, die Sozialpolitik betraf und dennoch jede Vertragsverhandlung von Rom bis Amsterdam gerade in der Frage der sozialpolitischen Kompetenzausstattung der Union ihre konfliktträchtigsten Auseinandersetzungen hatte. ${ }^{3}$ Diese waren oft nur mit mühsamen Kompromissen (darunter etwa das britische ,opting-out ${ }^{\text {* }}$ im Maastricht-Vertrag) zu lösen. Auch in der Ratifikationskrise des Verfassungsvertrages nach dem französischen ,Nein' flackerte, wie zu beleuchten sein wird, die Diskussion um eine ,Nachjustierung' der sozialen Dimension auf, um freilich bald in den Mühlen schwieriger Kompromissbildungsprozesse, die prioritär der institutionellen Machtarchitektur galten, klein gemahlen zu werden.

Die Sozialpolitik ist zweifellos eines der sensiblen Politikfelder, auf dem in den vertragspolitischen ,great bargains' der Regierungen grundlegende Zielkonflikte über Wesen und Gestalt der Union (wirtschaftlicher Zweckverband oder politische Union) ebenso zum Tragen kommen wie konkrete mitgliedstaatliche Souveränitätsvorbehalte. Diese sind ihrerseits Ausdruck unterschiedlicher, aus der ökonomischen und sozialstaatlichen Verfasstheit der Mitgliedstaaten resultierender gesellschaftlicher Präferenzen und beeinflussen die Bereitschaft zu einem ,wohlfahrtspolitischen Verantwortungstransfer nach Europa“. 4

Unter Zuhilfenahme dieser Vorüberlegungen lässt sich die vertragspolitische Entwicklung wie folgt skizzieren: Die Ökonomie war und ist Kernbereich und strategische Entwicklungsachse der europäischen Integration. Der Vorrang der Ökonomie manifestiert sich im Integrationsverlauf über die Stufen des Gemeinsamen Marktes (EWG-Vertrag), des Binnenmarktes (Einheitliche Europäische Akte) und der Währungsunion (Maastrichter Vertrag). Jede Stufe der Marktintegration führt jedoch zu je spezifischen Auseinandersetzungen um die Reichweite, die Kompetenzstrukturen und Entscheidungsmodi einer der Marktintegration ,gemäßen“ gemeinschaftlichen Sozialpolitik. Ohne die asymmetrische Grundarchitektur zu überwinden wurden im Laufe der Integrationsentwicklung die legislativen Zuständigkeiten der Europäischen Union in den Bereichen der Arbeits-, Beschäftigungs- und Sozialpolitik sukzessive erweitert und die Entscheidungsregeln wiederholt angepasst. Legt man die Ergebnisse der vertragspolitischen, great bargains" zugrunde, so sind die jeweiligen Vertragsgrundlagen - ausgehend von der schmalen Basis des EWG-Vertrages (Regelungskompetenzen für Wanderarbeitnehmer, Sozialfonds) - stufenweise erweitert worden. Der Schritt vom EWG-Vertrag zur Einheitlichen Europäischen Akte (EEA) war graduell, der von der EEA zum Maastricht-Vertrag substanziell, der des Amsterdamer Vertrages wiederum graduell und der des Nizza-Vertrages minimal.

Im Einzelnen betrachtet, hat sich die EU-Sozialpolitik erst nach dem Pariser Gipfel von 1972 mit einem sozialpolitischen Aktionsprogramm als eigenständiges Politikfeld etabliert. Die EEA schuf vertragliche Grundlagen insbesondere für Maßnahmen im Bereich Sicherheit und Gesundheitsschutz am Arbeitsplatz, die mit qualifizierter Mehrheit verabschiedet werden konnten. Der Maastrichter Vertrag erweiterte die Zuständigkeit der Europäischen Union unter

3 Hans-Wolfgang Platzer: Deutschland und die europäische Sozial- und Beschäftigungspolitik - Determinanten, Entwicklungen, Perspektiven, in: Heinrich Schneider/Mathias Jopp/Uwe Schmalz (Hrsg.): Eine neue deutsche Europapolitik? Rahmenbedingungen - Problemfelder - Optionen, Bonn 2001, S. 421-448.

4 Steffen Mau: Wohlfahrtspolitischer Verantwortungstransfer nach Europa? Präferenzstrukturen und ihre Determinanten in der europäischen Bevölkerung, in: Zeitschrift für Soziologie 4/2003, S. 302-324. 
anderem in den Bereichen Arbeitsbedingungen und Geschlechtergleichbehandlung (mit qualifizierten Mehrheitsentscheidungen) sowie soziale Sicherheit. Letzteres Politikfeld unterliegt der Einstimmigkeit. Die damit verbundenen Veto-Konstellationen erklären die weitgehende Stagnation einer regulativen Politik in diesem Bereich und ,policy-outputs', die sich allenfalls auf der Ebene unverbindlicher Rechtsakte bewegen. Dieser vertragspolitische Erfahrungshintergrund verweist zugleich auf die jüngeren Versuche einer sozialpolitischen Problembearbeitung mittels der Steuerungsform der Offenen Methode der Koordinierung (OMK). ${ }^{5}$ Der Amsterdamer Vertrag verankerte die Beschäftigungspolitik als Gemeinschaftsaufgabe, wobei die nach schwierigen Vertragsverhandlungen zur EU-Beschäftigungspolitik gefundenen institutionellen und prozeduralen Kompromisse ${ }^{6}$ zugleich die Grundlogik der OMK konstituierten und er führte nach dem Ende des britischen ,opting-out ' zur Integration des Maastrichter sozialpolitischen Protokolls ${ }^{7}$ in das Vertragswerk. Schließlich ergänzte der Vertrag von Nizza den EU-Kompetenzkatalog um den Bereich Antidiskriminierung.

Wirft man einen kurzen Blick auf den sozialpolitischen acquis communautaire, der sich bisher unter diesen vertraglichen Rahmenbedingungen herausgebildet hat, ${ }^{8}$ so reflektiert dieser zunächst in starkem Maße wettbewerbs- und mobilitätsbezogene Funktionserfordernisse. Entsprechende Regulierungen, die (im weitesten Sinne) auf dem Prinzip der Harmonisierung basieren, sind demzufolge in den Bereichen der sozialen Sicherheit der Wanderarbeitnehmer, der Sicherheit und dem Gesundheitsschutz am Arbeitsplatz, der beruflichen Gleichbehandlung von Männern und Frauen und im Bereich des individuellen Arbeitsrechts (zum Beispiel Mindestschutz bei Massenentlassungen und Betriebsübergang) anzutreffen. Diese nach der ,Gemeinschaftsmethode" erlassenen Richtlinien beziehungsweise Verordnungen variieren in ihrem Regulierungsniveau, wobei einerseits - vor allem im Zuge der Binnenmarktregulierung - das Prinzip der Mindeststandards gilt, andererseits, wie etwa im Bereich des Arbeits- und Gesundheitsschutzes, das Niveau und die Innovationskraft der Gemeinschaftsgesetzgebung als hoch bewertet werden kann. ${ }^{9}$ Die eingangs theoretisch hergeleitete Annahme, dass sich im Vergleich zu produkt- und mobilitätsbezogenen Regelungen die Regulierung produktions- und standortgebundener Materien als schwierig herausstellt, unterstreichen die Erfahrungen im Bereich des kollektiven Arbeitsrechts. Hier blieben zwei Gesetzgebungsvorhaben - die Regelung der Information und Konsultation von Arbeitnehmern in transnationalen Unternehmen und die Mitbestimmung in der ,Europäischen Aktiengesellschaft ${ }^{\star}$ - über Jahrzehnte blockiert. Es bedurfte neben der im Maastrichter Vertrag für diese Regelungsmaterien eingeführten Mehrheitsentscheidung vor allem eines - von der Europäischen Kommission klug orchestrierten - Paradigmenwechsels im Regulierungsansatz, um diese Gesetzgebungsvorhaben zu realisieren. Dabei

5 Stamati Devetzi/Hans-Wolfgang Platzer (Hrsg.): Offene Methode der Koordinierung und Europäisches Sozialmodell. Interdisziplinäre Perspektiven, Stuttgart im Erscheinen.

6 Hans-Wolfgang Platzer: Die EU-Sozial- und Beschäftigungspolitik nach Amsterdam: Koordinierte und verhandelte Europäisierung?, in: integration 3/1999, S. 176-190.

7 Protokoll über die Sozialpolitik zum Vertrag über die Europäische Union, in: Amtsblatt der EG, Nr. C 191 vom 29. Juli 1992, S. 90.

8 Vgl. Bernd Schulte: Die Entwicklung der Sozialpolitik der Europäischen Union und ihr Beitrag zur Konstituierung des europäischen Sozialmodells, in: Hartmut Kaelble/Günther Schmid (Hrsg.): Das europäische Sozialmodell. Auf dem Weg zum transnationalen Sozialstaat. WZB-Jahrbuch 2004, Berlin 2004, S. 75-103; HansWolfgang Platzer: EU-Mehrebenensystem und „Europäisches Sozialmodell““, in: Matthias Chardon/Ursula Göth/Martin Große Hüttmann/Christine Probst-Dobler (Hrsg.): Regieren unter neuen Herausforderungen: Deutschland und Europa im 21. Jahrhundert, Baden-Baden 2003, S. 221-236.

9 Volker Eichener/Helmut Voelzkow: Europäische Regulierung im Arbeitsschutz. Überraschungen aus Brüssel und ein erster Versuch ihrer Erklärung, in: Volker Eichener/Helmut Voelzkow (Hrsg.): Europäische Integration und verbandliche Interessenvermittlung, Marburg 1994, S. 385-417. 
schuf die Richtlinie zur Einrichtung Europäischer Betriebsräte ${ }^{10}$ (EBR) vom September 1994 erstmals eine Institution des kollektiven Arbeitsrechts auf europäischer Ebene. Der Regulierungsmodus der EBR-Richtlinie ist insbesondere dadurch gekennzeichnet, dass an die Stelle substanzieller Normen und materieller Regeln detaillierte Verfahrensregeln (Prozeduralisierung) treten und eine (früher stets erfolglose) harmonisierende Rahmenregelung durch das Prinzip der Optionalisierung also durch unternehmensspezifische Aushandlungsprozesse ersetzt wird. Die paradigmatische Bedeutung dieses Regulierungsansatzes, der als „regulierte Selbstregulierung"11 gekennzeichnet werden kann, wird auch dadurch unterstrichen, dass sich die 2001 verabschiedete Richtlinie über die Arbeitnehmermitbeteiligung in der ,Europäischen Aktiengesellschaft ${ }^{t 12}$ ebenfalls stark an diesem Modus orientiert.

Ein gewichtiges institutionelles Element der sozialpolitischen EU-Governance ist seit dem Maastrichter Vertrag der (multi-)sektorale Soziale Dialog zwischen den europäischen Sozialpartnern, deren Verhandlungen und Vereinbarungen den sozialpolitischen Besitzstand der Europäischen Union gleichfalls angereichert haben. Zu den Ergebnissen des Sozialen Dialogs, die anschließend durch Ministerratsbeschluss in EU-Recht umgesetzt wurden (das Verfahren der sogenannten, verhandelten Gesetzgebung'), zählen die Vereinbarungen zum Elternurlaub (1996), zur Teilzeitarbeit (1997) und zu befristeten Arbeitsverträgen (1999).

Die seit dem Maastrichter Vertrag gleichfalls mögliche tarifautonome Option einer supranationalen ,Selbstregulierung ' arbeits- und tarifpolitischer Gegenstände durch die Sozialpartner kam nahezu ein Jahrzehnt nicht zum Tragen. Erst 2002 konnte ein erstes Rahmenabkommen zur Telearbeit abgeschlossen werden, dem eine Rahmenvereinbarung zum „Lebenslangen Lernen“ (2002) und zum „Stress am Arbeitsplatz“ (2004) folgten. Schließlich konnten 2005 ein „Aktionsrahmen zur Gleichstellung von Frauen und Männern“ und 2007 eine Vereinbarung über „Belästigung und Gewalt am Arbeitsplatz“ vereinbart werden. Diese autonomen Rahmenabkommen, die bislang primär, weichen ‘ Materien gelten, bedürfen der Implementierung durch die nationalen Gewerkschaften und Arbeitgeber vermittels der nationalstaatlichen Praxen der Arbeitsbeziehungen. Demgegenüber wird die oben genannte , verhandelte Gesetzgebung' durch eine Richtlinie des Rats umgesetzt und damit, allgemeinverbindlich'.

Dieser Modus der autonomen Selbstregulierung wirft insbesondere bei der Implementierung zahlreiche Probleme auf. Neben der Durchführungsbereitschaft der Sozialpartner (compliance) können auch die Strukturen und die Leistungsfähigkeit der nationalen Arbeitsbeziehungssysteme eine effektive Umsetzung und Anwendung der europäischen Rahmenvereinbarung auf den jeweils dezentralen oder lokalen Ebenen erschweren oder verhindern. Leiber und Schäfer charakterisieren diese jüngere Entwicklungstendenz des Sozialen Dialogs als „,doppelten Voluntarismus“, wonach ,[...] zur Verlagerung von Regelungskompetenz auf freiwillige Vereinbarungen durch die Sozialpartner (prozedurale Ebene), der empfehlende, unverbindliche Modus bei der Umsetzung dieser Vereinbarungen (inhaltliche Ebene) tritt". 13

10 Richtlinie 94/45/EG des Rates vom 22. September 1994 über die Einsetzung eines Europäischen Betriebsrats oder die Schaffung eines Verfahrens zur Unterrichtung und Anhörung der Arbeitnehmer in gemeinschaftsweit operierenden Unternehmen und Unternehmensgruppen, in: Amtsblatt der EG, Nr. L 254 vom 30. September 1994, S. 64-72.

11 Hans-Wolfgang Platzer: Europäisierung und Transnationalisierung der Arbeitsbeziehungen in der EU, in: Internationale Politik und Gesellschaft 2/2002, S. 103-121.

12 Richtlinie 2001/86/EG des Rates vom 8. Oktober 2001 zur Ergänzung des Statuts der Europäischen Gesellschaft hinsichtlich der Beteiligung der Arbeitnehmer, in: Amtsblatt der EG, Nr. L 294 vom 10. November 2001, S. 22-32.

13 Simone Leiber/Armin Schäfer: Der doppelte Voluntarismus in der EU-Sozial- und Beschäftigungspolitik, in: Ingeborg Tömmel (Hrsg.): Die Europäische Union. Governance und Policy-Making, Politische Vierteljahresschrift Sonderheft 40/2007, S. 116-135, hier S. 117. 
Für eine Gesamtbewertung der ,policy-outputs“ im Bereich einer sozial-regulativen EUPolitik sind schließlich die Befunde einer quantitativen Untersuchung von Bähr, Treib und Falkner sehr aufschlussreich, die sich der Entwicklung bindender und nicht-bindender Rechtsakte in der EU-Sozialpolitik (im Vergleich mit der Umweltpolitik) im Zeitraum 1970 bis 2004 widmet. Demnach nimmt seit der Institutionalisierung der beiden Politikfelder in den 1970er Jahren die Zahl sowohl der bindenden als auch der nicht-bindenden Rechtsakte in beiden Bereichen kontinuierlich zu. In beiden Politikbereichen übersteigt die Zahl der bindenden Rechtsakte die Anzahl an unverbindlichen Regelungen. Während bei den verbindlichen Rechtsakten (bis 2004) die Umweltpolitik mit insgesamt knapp 250 Regelungen deutlich vor der Sozialpolitik mit insgesamt 155 bindenden Rechtsakten liegt, ist es im Bereich der nicht-bindenden Regelungen umgekehrt; hier liegt die Zahl in der Sozialpolitik bei rund 150 und in der Umweltpolitik bei circa 75. ${ }^{14}$ Auch bei einem genaueren Blick auf die einzelnen Regelungen bekräftigen sich diese numerischen Relationen und Trends: „Zum einen verstärkt sich die quantitative Relevanz nicht-bindender Regelungen in der EU-Sozialpolitik sogar noch, wenn Änderungen, Anwendungsregeln und geografische Ausdehnung, die in der Form von bindenden Rechtsakten verabschiedet werden, von Verordnungen und Richtlinien unterschieden werden, die einen neuen Regelungsbereich zum Inhalt haben. In der Sozialpolitik liegt zu keinem Zeitpunkt während des gesamten Untersuchungszeitraums die Zahl der nicht-bindenden Regelungen unter der Zahl der bindenden Rechtsakte. Seit Anfang der 1980er Jahre ist sogar ein verstärkter Anstieg unverbindlicher Maßnahmen zu beobachten." 15

Zur Erklärung der empirischen Befunde ziehen Bähr et alii institutionelle Faktoren (Zugangsregeln und Entscheidungsregeln) und die Präferenzen der an der Entscheidung beteiligten Akteure heran. Dabei gehen sie davon aus, dass trotz der Agenda-Setting-Macht der Kommission und der zunehmenden Bedeutung des Europäischen Parlaments im Gesetzgebungsverfahren es nach wie vor die Positionen der Regierungen sind, von denen die Einigungschancen im Rat und das Ob und Wie europäischer Gesetzgebung abhängen. ${ }^{16}$ Die Regierungspräferenzen in europäischen Verhandlungen sind wiederum durch ökonomische und institutionelle Kosten-Nutzen-Kalküle sowie durch parteipolitische Ideologien einschließlich der Befriedigung von Klientelinteressen beeinflusst. Im hier interessierenden Zusammenhang sind die folgenden Erklärungen, die Bähr et alii anbieten, bedeutsam. In der Sozialpolitik ist (stärker als in der Umweltpolitik) ein deutlicher vertraglich-institutioneller Effekt dergestalt zu beobachten, dass bindende Rechtsakte nur dann und nur in den ,issue areas ' verabschiedet werden, in denen die Europäische Union eine explizite Zuständigkeit besitzt und Entscheidungen mit qualifizierter Mehrheit getroffen werden. In anderen , issue areas ' der Sozialpolitik (Familie, ältere Personen, Behinderte etc.) sind nahezu ausschließlich nicht-bindende Regelungen anzutreffen.

Auch die Bedeutung ökonomischer und institutioneller Kosten unterschiedlicher Regulierungsarten und -materien wird dahingehend unterstrichen, dass produktbezogene Regelungen, die in der Regel weniger konfliktgeladen sind, in der Umweltpolitik eine größere Rolle spielen (und unter anderem die höhere Zahl verbindlicher Rechtsakte erklären) als in der Sozialpolitik, bei der Regulierungsvorhaben häufiger eine produktionsbezogene Dimension haben: „Produktbezogene Regelungen, [...] können zwar erhebliche [...] Kosten verur-

14 Holger Bähr/Oliver Treib/Gerda Falkner: Von Hierarchie zu Kooperation? Zur Entwicklung von GovernanceFormen in zwei regulativen Politikfeldern der EU, in: Ingeborg Tömmel (Hrsg.): Die Europäische Union. Governance und Policy-Making, Politische Vierteljahresschrift Sonderheft 40/2007, S. 92-115, hier S. 97 ff.

15 Ebenda, S. 98.

16 Ebenda, S. 101. 
sachen. Zugleich aber haben sowohl die Mitgliedstaaten als auch die betroffenen Produzenten ein gemeinsames Interesse an europaweit gültigen Standards, weil diese dann den Handel mit diesen Produkten im Binnenmarkt erleichtern. Insofern sind solche Regelungen leichter zu verabschieden als produktionsbezogene Standards, die den Unternehmen Kosten auferlegen, ohne dass dadurch nennenswerte Vorteile in Bezug auf die Vermarktbarkeit von Produkten entstehen. " 17 Ein weiterer gewichtiger Erklärungsfaktor ist die unterschiedlich starke Verwurzelung der beiden Politikbereiche in den nationalen Politiktraditionen. Im Gegensatz zur Sozialpolitik, deren Institutionalisierung in vielen Ländern mit der Konsolidierung des Nationalstaates einherging und tief in den nationalen Traditionen verankert ist, bildete sich die Umweltpolitik als eigenständiges Politikfeld in den Mitgliedstaaten erst ab den 1970er Jahren. Demzufolge konnte sich die gleichzeitig begonnene EU-Umweltpolitik gegenüber einem relativ schwach institutionalisierten Politikfeld in den Mitgliedstaaten leichter behaupten und (im Vergleich zur Sozialpolitik) stärker entwickeln. Schließlich erklärt die unterschiedliche Akzeptanz europäischer Regulierung in den nationalen Wählerschaften die unterschiedlichen , policy-outputs ' zwischen den beiden Politikbereichen. Bereits Eurobarometer-Daten zeigen, dass eine Mehrheit der Bürgerinnen und Bürger in den Mitgliedstaaten die Europäische Union als geeignete Ebene zur Lösung von Umweltproblemen betrachtet und nur eine Minderheit die nationalstaatliche Ebene, während es bei der Sozialpolitik umgekehrt ist. Auch wenn es, so Bähr et alii, ,,[... in der alltäglichen Entscheidungsfindung häufig keinen direkten Bezug zwischen Regierungen und ihren Wählerinnen und Wählern gibt, so weist die beobachtete Asymmetrie doch darauf hin, dass es für Regierungen in der Umweltpolitik leichter ist, gemeinsame europäische Regelungen gegenüber ihrer nationalen Wählerschaft zu vertreten, als dies im Bereich der Sozialpolitik der Fall ist" ${ }^{\text {" }}{ }^{18}$

Die Schwierigkeiten einer primärrechtlichen Erweiterung der sozialpolitischen Handlungsgrundlagen und einer sekundärrechtlichen Ausgestaltung der sozialen Dimension sind schließlich auch darauf zurückzuführen, dass machtvolle gesellschaftliche Akteursgruppen, wie die Gewerkschaften, die in ihren nationalen ordnungspolitischen Traditionen einer sozial-regulativen Politik verpflichtet sind, aus dieser gemeinsamen sozial- und wirtschaftspolitischen Grundhaltung heraus keineswegs durchweg gleichgerichtete integrationspolitische Zielvorstellungen und suprastaatliche Problemlösungsstrategien verfolgen. Eine Untersuchung von Busemeyer et alii, ${ }^{19}$ die mit Blick auf die wirtschafts- und sozialpolitischen Handlungsfelder des europäischen Sozialmodells eine ,europäische Landkarte gewerkschaftlicher Interessen“ vermisst, kommt zu folgenden Ergebnissen:

Die ,preference formation“ ${ }^{20}$ bezüglich der europäischen Handlungsebene ist in starkem Maße von der gewerkschaftlichen Einbettung in das jeweilige nationale Sozialstaats- und Arbeitsbeziehungsmodell und der darin institutionell verankerten und entfaltbaren relativen Gewerkschaftsmacht abhängig. Diese nationalen ,customs and practices“ prägen nachhaltig die Vorstellungen zum Auf- und Ausbau der europäischen Handlungsebene. Entsprechend formieren sich die EU-bezogenen Interessen der nationalen Gewerkschaften in nach Themenfeldern variierenden Clustern, wobei folgende Grundmuster sichtbar werden:

Zum einen zeigt sich, dass je stärker in einem konkreten Handlungsfeld die nationale Interessenvermittlungsfähigkeit gegeben ist beziehungsweise je positiver sie bewertet wird

17 Ebenda, S. 108.

18 Ebenda, S. 111.

19 Marius R. Busemeyer/Christian Kellermann/Alexander Petring/Andrej Stuchlik: Overstretching Solidarity? Trade Unions" National Perspectives on the European Economic and Social Model, in: Friedrich Ebert Stiftung (Hrsg.): Internationale Politikanalyse, Bonn/Berlin 2007.

20 Ebenda, S. 3. 
(etwa seitens der skandinavischen Gewerkschaften) desto geringer ist der artikulierte Bedarf an und die politische Bereitschaft zu europäischen Politikansätzen. Ein umgekehrtes Muster findet sich bei den national eher ,schwächeren' Gewerkschaften (etwa innerhalb des ,mediterranen` Typs), die einer Stärkung europäischer Institutionen, Regelungen und Verfahren offener gegenüberstehen beziehungsweise diese nachdrücklich präferieren. Zum anderen zeigt sich, dass je allgemeiner die europäischen Politikziele formuliert sind (,Stärkung der sozialen Dimension des EU-Binnenmarktes“) desto höher ist die Konvergenz und Schnittmenge der Interessen; je konkreter jedoch die Frage nach den auf europäischer Ebene zu entwickelnden Politikinstrumenten gestellt wird, desto divergierender sind die Politikoptionen und desto geringer ist die jeweilige Schnittmenge zwischen den einzelnen Gewerkschaften oder, Gewerkschaftsfamilien`.

\section{Vom Konvent über den Verfassungsvertrag zum Reformvertrag von Lissabon: Ent- wicklungslinien, Interessenkonstellationen und Ergebnisse}

Unter den ,left-overs' des Vertrages von Nizza (Vereinfachung des Vertragswerks, Kompetenzabgrenzung zwischen den Mitgliedstaaten und der europäischen Ebene, rechtlicher Status der Grundrechtscharta, Demokratisierung der Europäischen Union und Beteiligung der nationalen Parlamente) und den in der „Erklärung von Laeken“ vom Dezember 2001 formulierten Reformaufträgen an den Konvent taucht die Sozialpolitik als eigenständiger Reformkomplex nicht auf. Sie war hingegen ,mittelbar" durch die in der Grundrechtscharta enthaltenen sozialpolitischen Bestimmungen von Anfang an Teil der Konvents-Agenda.

In den Konventsberatungen wurden sozialpolitische Aspekte zunächst in der Arbeitsgruppe „Ordnungspolitik“ mitverhandelt. Erst als sich bei einer Aussprache im Plenum im November 2002 zahlreiche Konventsmitglieder kritisch über das Fehlen sozialpolitischer Reformvorschläge äußerten, wurde schließlich eine Arbeitsgruppe „Soziales Europa“ eingerichtet, die sich sowohl mit der Frage beschäftigen sollte, welche sozialen Werte und Ziele in den Vertrag aufgenommen werden sollten, als auch mit der Frage einer Veränderung der Kompetenzen und Entscheidungsverfahren. Eine detaillierte Analyse des Konventsprozesses von Treib zeigt zunächst, ${ }^{21}$ dass in dieser Arbeitsgruppe eine klare Mehrheit für eine zumindest moderate Ausweitung der Kompetenzen und Mehrheitsentscheidungen plädierte. Sie zeigt ferner, dass in den einzelnen Voten der Ausschussmitglieder, wie zu erwarten, Ländermuster sichtbar wurden (wobei sich zu den ,traditionellen Skeptikern' Großbritannien und Irland, auch zwei neue Mitgliedstaaten, Tschechien und Estland gesellten, die durch eine Ausweitung sozialpolitischer EU-Regelungen nationale Anpassungskosten befürchteten), dass aber letztlich die (partei-)politische Verankerung entlang des Links-Rechts-Spektrums die jeweiligen Haltungen bezüglich einer deutlichen Ausweitung, einer moderaten Stärkung oder keiner Veränderung des vertraglichen Status quo prägte. Die Grundlinie des Ausschusses, die soziale Dimension vertragspolitisch moderat auszubauen, war jenseits der erfolgreichen Aufnahme der Grundrechtscharta in den Korpus des Vertrages und einer Präzisierung und Stärkung sozialer Werte und Ziele in den allgemeinen Vertragsbestimmungen im letztlich entscheidenden ,materiellen“ Bereich der Kompetenzzuweisungen und Entscheidungsverfahren in den Abschlussberatungen des Konvents nicht mehrheitsfähig. Hier waren die ,roten Linien', die von den Regierungsvertretern Großbritanniens, Irlands, Spaniens, Tschechiens und Estlands gezogen wurden, so deutlich und deren Widerstand so groß, „,dass am Ende keine andere Lösung möglich war, als den Bereich der Kompetenzen und

21 Oliver Treib: Der EU-Verfassungsvertrag und die Zukunft des Wohlfahrtsstaates in Europa, in: Reihe Politikwissenschaft Nr. 99 des Instituts für Höhere Studien, Wien 2004. 
Mehrheitsentscheidungen im Verfassungsentwurf bis auf den durch die legislative ,Notbremse' abgeschwächten Übergang zu Mehrheitsentscheidungen bei der sozialen Sicherheit der Wanderarbeitnehmer so zu belassen wie bisher". ${ }^{22}$ Die Aufnahme einer auf belgischem Vorschlag beruhenden sogenannten ,horizontalen Sozialklausel ‘ fand im Konvent zunächst keine Mehrheit und wurde erst in der anschließenden Regierungskonferenz (in leicht abgeschwächter Form) in den Verfassungsvertrag aufgenommen.

Der sozialpolitisch dergestalt ausgestattete Verfassungsvertrag mit seinen Neuerungen einer Stärkung sozialer Werte und Ziele und einer (im dritten Teil des Vertrages) in der Substanz unveränderten sozial-ökonomischen Kompetenz- und Entscheidungsorganisation fand im Ratifizierungsprozess mit dem ,Nein' in den Referenden in Frankreich und den Niederlanden sein jähes Ende.

Wie Analysen der innenpolitischen Auseinandersetzungen und des Wahlverhaltens in beiden Ländern zeigen, waren die Abstimmungsergebnisse jeweils einer komplexen Gemengelage aus europa- und innenpolitischen Motiven, Interessen und Kalkülen geschuldet, wobei in Frankreich - weit stärker als in den Niederlanden - der hier interessierende Themenkreis eine zentrale Rolle spielte: „Wichtiger als die Trennlinie nationale Souveränität und supranationale EU-Integration war [...] eine Links-Rechts-Trennlinie in Gestalt eines Wettbewerbs zwischen der Konzeption eines ,neoliberalen' und eines ,sozialen` Europas. Hier wurde um mehr Markt oder mehr ,Staat' (im Sinne einer supranationalen Politikgestaltung auf EU-Ebene) gerungen, nicht um das Verhältnis zwischen Nation und Europa [...] Der normative Maßstab für die linke Kritik am Verfassungsvertrag war das Modell einer Union, die dank Mehrheitsentscheidungen im Rat soziale Mindeststandards und eine Mindestbesteuerung definieren und damit, Sozialdumping ' und einen ungezügelten Wettbewerb in der Besteuerung mobilen Kapitals verhindern kann und zugleich ihre Fähigkeit zur wachstumsfördernden wirtschaftspolitischen Koordinierung als Gegengewicht zur Europäischen Zentralbank stärkt." 23

Vor diesem Hintergrund war in der sogenannten ,Reflexionsphase', die sich die Europäische Union nach dem Scheitern des Verfassungsvertrages verordnete, auch das Thema der EU-Sozialpolitik - zumindest kurzzeitig - repolitisiert. Zu den in Wissenschaft und Politik diskutierten Optionen zählten die Neuformulierung einzelner Verfassungsartikel, die Abtrennung des dritten Teils der Verfassung, die Ergänzung der Verfassung durch ein Sozialprotokoll und eine von den Regierungen zu beschließende politische Erklärung zum sozialen Europa.

Die maßgeblich unter deutscher Ratspräsidentschaft gestellten Weichen, ${ }^{24}$ die schließlich zum Vertrag von Lissabon führten, sahen vor, rasch ein runderneuertes Vertragswerk auszuarbeiten, das auf den Verfassungsbegriff (und entsprechende Symbole) verzichtet, auf der Struktur bisheriger Verträge aufbaut und die Substanz der im Verfassungsvertrag erzielten Kompromisse nicht dadurch gefährdet, dass das ,Gesamtpaket' durch die erneute Verhandlung von Sachpolitiken und politikbereichsspezifischen Einzelfragen aufgeschnürt würde. Damit waren auch die Optionen einer sozialpolitischen Nachjustierung begrenzt und insbesondere eine mögliche Erweiterung der , materiellen' Kompetenzen und Entscheidungsinstrumente (im dritten Teil des Verfassungsvertrages) letztlich ausgeklammert. Unter den rund

22 Ebenda, S. 26.

23 Joachim Schild: Ein Sieg der Angst - das gescheiterte französische Verfassungsreferendum, in: integration 3/2005, S. 187-200, hier S. 193.

24 Zur ,road map' siehe Wolfgang Wessels/Anne Faber: Vom Verfassungsvertrag zurück zur ,Methode Monnet'? Die Entstehung der ,Road Map‘ zum EU-Reformvertrag unter deutscher Ratspräsidentschaft, in: integration 4/2007, S. 370-381. 
zwölf sogenannten „focal points“25 der Sondierungen und Verhandlungen der Regierungen befand sich dementsprechend nur die Option einer allgemeinen Bekräftigung oder stärkeren (symbolischen) Betonung der sozialen Dimension der Europäischen Union im Vertrag. Hierbei zählten wiederum nur Frankreich, Deutschland, Belgien und die SPE-Fraktion im Europäischen Parlament zu den Protagonisten dieser Option, wobei Frankreich nach der Präsidentschaftswahl diesen Verhandlungspunkt nicht weiterverfolgte. ${ }^{26}$

Im Gesamtergebnis des Lissabonner Vertragswerkes kommt diese Option nicht zum Tragen. Vielmehr erfuhr der im Verfassungsvertrag erzielte vertragspolitische acquis dahingehend noch eine Schwächung, dass Großbritannien und Polen die äußerst komplexe und schwierige Verhandlungskonstellation dazu nutzten, sich im Hinblick auf den Geltungsbereich und Durchsetzungsmodus der Grundrechtscharta ein ,opting-out‘ einzuräumen.

\begin{tabular}{|c|c|c|c|}
\hline $\begin{array}{l}\text { Vertragsbasis } \\
\quad \text { (Nizza) }\end{array}$ & Regelungsgegenstand & Neuerung & $\begin{array}{l}\text { Vertragsbasis } \\
\text { (Lissabon) }\end{array}$ \\
\hline EUV & \multicolumn{2}{|c|}{ Titel I - Gemeinsame Bestimmungen } & EUV \\
\hline Art. 2 & Zielsetzungen & $\begin{array}{l}\text { Stärkung sozialer Werte und } \\
\text { Ziele }\end{array}$ & Art. 3 \\
\hline Art. 6 & Grundrechte & $\begin{array}{l}\text { ergänzt um die Rechtsverbind- } \\
\text { lichkeit der Charta der Grund- } \\
\text { rechte der Europäischen Union. } \\
\text { Polen und Großbritannien ver- } \\
\text { fügen aber über, opting-outs'. }\end{array}$ & Art. 6 \\
\hline EGV & \multicolumn{2}{|c|}{ Erster Teil, Titel II - Allgemein geltende Bestimmungen } & AEUV \\
\hline \multirow[t]{2}{*}{-} & ,Horizontale Sozialklausel’ & neu eingeführt & Art. 9 \\
\hline & \multicolumn{2}{|c|}{$\begin{array}{l}\text { Dritter Teil, Titel IV - Die Freizügigkeit, } \\
\text { der freie Dienstleistungs- und Kapitalverkehr }\end{array}$} & \\
\hline \multirow[t]{2}{*}{ Art. 42} & $\begin{array}{l}\text { Soziale Sicherheit bei Wander- } \\
\text { arbeitnehmern }\end{array}$ & $\begin{array}{l}\text { Einführung einer sogenannten } \\
\text { Notbremse mit aufschiebender } \\
\text { Wirkung }\end{array}$ & Art. 48 \\
\hline & \multicolumn{2}{|c|}{ Dritter Teil, Titel IX - Beschäftigung } & \\
\hline \multirow[t]{2}{*}{ Art. $125-130$} & $\begin{array}{l}\text { Bestimmungen hinsichtlich ei- } \\
\text { ner koordinierten Beschäfti- } \\
\text { gungsstrategie bzw. Koordinie- } \\
\text { rung der Beschäftigungspolitik }\end{array}$ & & Art. $145-150$ \\
\hline & \multicolumn{2}{|c|}{ Dritter Teil, Titel X-Sozialpolitik } & \\
\hline Art. 136-145 & $\begin{array}{l}\text { Bestimmungen hinsichtlich der } \\
\text { Sozialpolitik }\end{array}$ & & Art. 151-161 \\
\hline
\end{tabular}

25 Andreas Maurer: Verhandlungen zum Reformvertrag unter deutschem Vorsitz, in: Aus Politik und Zeitgeschichte 43/2007, S. 3-8, hier S. 6.

26 Ebenda. 


\begin{tabular}{|c|c|c|c|}
\hline $\begin{array}{l}\text { Vertragsbasis } \\
\text { (Nizza) }\end{array}$ & Regelungsgegenstand & Neuerung & $\begin{array}{l}\text { Vertragsbasis } \\
\text { (Lissabon) }\end{array}$ \\
\hline- & Sozialgipfel & $\begin{array}{l}\text { erstmalige primärrechtliche } \\
\text { Verankerung }\end{array}$ & Art. 152 \\
\hline \multirow[t]{2}{*}{ Art. 140} & $\begin{array}{l}\text { Bestimmungen hinsichtlich der } \\
\text { Zusammenarbeit der Mitglied- } \\
\text { staaten in bestimmten Berei- } \\
\text { chen der Sozialpolitik }\end{array}$ & $\begin{array}{l}\text { Präzisierung und Fixierung des } \\
\text { Verfahrens }\end{array}$ & Art. 156 \\
\hline & \multicolumn{2}{|c|}{ Dritter Teil, Titel XI - Der Europäische Sozialfonds } & \\
\hline \multirow[t]{2}{*}{ Art. $146-148$} & $\begin{array}{l}\text { Bestimmungen hinsichtlich des } \\
\text { Europäischen Sozialfonds }\end{array}$ & & Art. $162-164$ \\
\hline & \multicolumn{2}{|c|}{ Protokolle } & \\
\hline \multirow[t]{2}{*}{-} & $\begin{array}{l}\text { Protokoll über die Anwendung } \\
\text { der Charta der Grundrechte der } \\
\text { Europäischen Union auf Polen } \\
\text { und das Vereinigte Königreich }\end{array}$ & neu hinzugefügt & Nr. 30 \\
\hline & \multicolumn{2}{|c|}{ Erklärungen } & \\
\hline- & $\begin{array}{l}\text { Erklärung zur Charta der } \\
\text { Grundrechte der Europäischen } \\
\text { Union }\end{array}$ & neu hinzugefügt & 1. Erklärung \\
\hline- & $\begin{array}{l}\text { Erklärung zu Artikel } 156 \text { des } \\
\text { Vertrags über die Arbeitsweise } \\
\text { der Europäischen Union }\end{array}$ & neu hinzugefügt & 31. Erklärung \\
\hline- & $\begin{array}{l}\text { Erklärung der Tschechischen } \\
\text { Republik zur Charta der Grund- } \\
\text { rechte der Europäischen Union }\end{array}$ & neu hinzugefügt & 53. Erklärung \\
\hline- & $\begin{array}{l}\text { Erklärung der Republik Polen } \\
\text { zur Charta der Grundrechte der } \\
\text { Europäischen Union }\end{array}$ & neu hinzugefügt & 61. Erklärung \\
\hline- & $\begin{array}{l}\text { Erklärung der Republik Polen } \\
\text { zu dem Protokoll über die An- } \\
\text { wendung der Charta der Grund- } \\
\text { rechte der Europäischen Union } \\
\text { auf Polen und das Vereinigte } \\
\text { Königreich }\end{array}$ & neu hinzugefügt & 62. Erklärung \\
\hline \multicolumn{4}{|c|}{$\begin{array}{l}\text { Quelle: Eigene Darstellung auf Grundlage des EUV und des EGV in den konsolidierten Fassungen des } \\
\text { Vertrages von Nizza, in: Amtsblatt der EU, Nr. C } 321 \text { vom 29. Dezember 2006, S. 1-331 und des EUV und } \\
\text { des AEUV in den konsolidierten Fassungen des Vertrages von Lissabon, in: Amtsblatt der EU, Nr. C } 115 \\
\text { vom 9. Mai 2008, S. 1-359. }\end{array}$} \\
\hline
\end{tabular}




\section{Die EU-Sozialpolitik im vertraglichen Reformprozess: die Sicht gesellschaftlicher Akteure}

EU-Vertragswerke sind komplexe Gebilde und Paketlösungen, die notwendigerweise immer mit Kompromissen behaftet sind. Dies macht es erforderlich, Gesamtbewertungen eines Vertragswerkes jenseits einzelner Politikfelder vorzunehmen. Eine solche Herangehensweise zeigt sich etwa in den Positionen des Europäischen Gewerkschaftsbundes (EGB) und des Deutschen Gewerkschaftsbundes (DGB) anlässlich der Verhandlungen zum Verfassungsvertrag. Beide Verbände begrüßten und unterstützen, trotz zum Teil weitergehender Forderungen und der Kritik an einzelnen Vertragselementen das Gesamtprojekt. ${ }^{27}$

Sie bewerten insbesondere die Rechtsverbindlichkeit der Grundrechtscharta und die verstärkte Verankerung des Sozialen Dialogs der Sozialpartner sowie des (neuen) „tripartiten Sozialgipfels für Wachstum und Beschäftigung“ positiv (vergleiche die Art. 152 und 156 des Vertrags über die Arbeitsweise der Europäischen Union in der konsolidierten Fassung des Lissabonner Vertrages, AEUV). Dieser bereits im Juli 2002 gleichsam extrakonstitutionell eingerichtete dreigliedrige Sozialgipfel soll die dreiseitigen Konsultationen zwischen den Sozialpartnern und Regierungen der Mitgliedstaaten ausbauen und die Sozialpartnerbeteiligung an den verschiedenen offenen Koordinierungsverfahren in den Bereichen Makroökonomie, Beschäftigung, Sozialschutz sowie allgemeine und berufliche Bildung verstetigen. Ferner sehen die gewerkschaftlichen Dachverbände in der Präzisierung sozialer Normen beziehungsweise der erstmaligen Verankerung der Ziele ,soziale Marktwirtschaft ${ }^{\circ}$, ,Vollbeschäftigung', ,soziale Gerechtigkeit', ,Solidarität zwischen den Generationen ' und ,Bekämpfung von sozialer Ausgrenzung und Diskriminierung' Fortschritte. In ähnlicher Weise die Gesamtergebnisse abwägend, kommt auch eine große Mehrheit der Parteien des Mitte-Links-Spektrums in der Europäischen Union zu zustimmenden Positionen sowohl zum Verfassungsvertrag wie zum Lissabon-Vertrag.

Wie oben gezeigt wurde (ohne dass dabei auf einzelne vertragliche Details eingegangen werden konnte), beinhaltet der Vertrag von Lissabon in seiner sozialpolitischen Kernsubstanz nicht mehr aber auch nicht weniger als der Konventsentwurf und der Vertrag über eine Verfassung für Europa. Ungeachtet dieser ,Kontinuität" haben sich inzwischen gleichwohl die Positionen innerhalb des europäischen Gewerkschaftslagers deutlich in Richtung einer dezidierten, die sozialpolitischen Lücken und Schwachstellen betonenden Kritik verschoben. Einzelne Bünde, darunter skandinavische Gewerkschaften, knüpfen aus nachstehend beschriebenen Gründen die weitere Unterstützung des Lissabonner Vertragsprozesses an Bedingungen und andere Verbände, wie etwa ver.di, stellen in jüngsten Positionspapieren ${ }^{28}$ die Gesamtarchitektur des sozial-ökonomischen Integrationsprozesses und den Verlauf des Reformprozesses auch unter demokratiepolitischen Vorzeichen infrage.

Diese gewerkschaftlichen Positionsveränderungen sind nicht zuletzt durch eine Reihe von Urteilen des Europäischen Gerichtshofes (unter anderem die Rechtssachen Laval, Viking, Rüffert ${ }^{29}$ ) in den Jahren 2007/2008 ausgelöst worden, die unter dem Vorrang der Marktfreiheiten des Binnenmarktes nationale Arbeitnehmer- und Gewerkschaftsrechte ein-

27 Deutscher Gewerkschaftsbund: Für eine europäische Verfassung. Stellungnahme des DGB zur EU-Regierungskonferenz, Beschluss des geschäftsführenden Bundesvorstandes vom 29.09.2003.

28 Ver.di: Einem sozialen Europa die Zukunft geben. Manifest zur Europapolitik - Grundzüge eines alternativen Wirtschafts- und Sozialmodells für die EU, unveröffentlichtes Manuskript des Bundesvorstandes, Berlin 2008.

29 Europäischer Gerichtshof: Rs. C-341/05 (Laval un Partneri/Svenska Byggnadsarbetareförbundet), Slg. 2007, I-11767; Europäischer Gerichtshof: Rs. C-438/05 (International Transport Workers ' Federation, Finnish Seamen's Union/Viking), Slg. 2007, I-10779; Europäischer Gerichtshof: Rs. C-346/06 (Rüffert/Land Niedersachsen), in: Amtsblatt der EU, Nr. C 128 vom 24. Mai 2008, S. 9. 
schränkten. Die Gewerkschaften sehen darin eine besorgniserregende Richtungsänderung und einen Bruch mit der bisherigen EU-Praxis, die nationale Souveränität des Arbeitsrechts und die Tarifvertragstraditionen zu respektieren. Diese Tendenz einer Unterordnung der sozialen Grundrechte unter die Dienstleistungsfreiheit sehen die Gewerkschaften auch durch den derzeit laufenden Revisionsprozess der Arbeitszeitrichtlinie bekräftigt, in dem erstmals in der Geschichte der Europäischen Union die Revision einer sozialpolitischen Richtlinie eine Verschlechterung des Arbeitnehmerschutzes mit sich bringen könnte. In Reaktion auf diese politischen Entwicklungen hat der EGB mittlerweile (Frühjahr 2008) eine Position entwickelt, die eine Ergänzung des Lissabonner Vertrages um eine ,Soziale Fortschrittsklausel' fordert. Dieses in Gestalt eines Vertrags-Protokolls detailliert ausformulierte „Protokoll zum Verhältnis zwischen wirtschaftlichen Freiheiten und sozialen Grundrechten im Lichte des sozialen Fortschritts "30 nimmt (das Inkrafttreten des Lissabon-Vertrages unterstellend) dementsprechend vertragstechnisch sowohl auf die entsprechenden Artikel des Vertrages über die Europäische Union (EUV) als auch auf den AEUV in den konsolidierten Fassungen des Lissabonner Vertrages Bezug. Organisationsintern mag diese EGB-Forderung einer vertraglichen Nachjustierung Ausdruck einer derzeit gerade noch konsensfähigen (Minimal-) Position bei zunehmend uneinheitlichen Zielvorstellungen einzelner Mitgliedsverbände sein; mit Blick auf die Ratifikationskrise des Lissabon-Vertrages und die oben analysierten Regierungspositionen mit ihren engen Manövrierspielräumen erscheint sie hingegen geradezu als ,Maximalforderung'. Betrachtet man neben den Gewerkschaften, die hier als, Seismografen ' der jüngsten Politisierungstendenzen in der Vertragsdebatte etwas ausführlicher behandelt wurden, die sonstigen sozialen Bewegungen und die Positionen der organisierten Zivilgesellschaft in Europa, so überwiegen etwa bei den Sozialverbänden und in der Umwelt- und Frauenbewegung die zustimmenden Positionen zum Reformvertrag. Demgegenüber lehnt das europäische Attac-Netzwerk, das bereits den Verfassungsvertrag abgelehnt hatte, konsequenterweise auch den Lissabon-Vertrag ab, weil auch in diesem das Problem „der Neoliberalismus erhält Verfassungsrang“ nicht gelöst sei. Die sich mit dem irischen ,Nein“ noch einmal wiederholende Ratifikationskrise erfordere vielmehr einen gänzlich neuen vertragspolitischen Aufbruch in Europa. ${ }^{31}$

\section{Bilanz und Perspektiven}

Vor dem Hintergrund der aktuellen politischen und gesellschaftlichen Debattenlandschaft war es das Ziel dieses Beitrags, die wesentlichen vertragspolitisch-konstitutionellen Entwicklungslinien aufzuzeigen. Zudem wurden die Ausprägungen und Ergebnisse der bisherigen sozialpolitischen EU-Governance skizziert, um auf dieser Grundlage Anhaltspunkte und Erklärungsmomente für den Verlauf und die Ergebnisse der vertragspolitischen Ausgestaltung des ,sozialen Europa‘ seit Nizza zu finden.

Die Analyse führt zum Befund eines ,konstitutionellen Minimalismus‘. Diese Bewertung ergibt sich aus dem in Grundzügen dargestellten Verhandlungsverlauf und den sozialpolitischen Ergebnissen, die durch zwei gegenläufige Tendenzen gekennzeichnet sind: Einerseits konnten zum Teil beachtliche Fortschritte im normativen Bereich erzielt werden; dazu zählen insbesondere die sozialpolitischen Zielbestimmungen der Union, die Rechtsverbindlichkeit der Grundrechtscharta (auch wenn sie nunmehr nur im Protokollanhang erscheint

30 Europäischer Gewerkschaftsbund: Protokoll zum Verhältnis zwischen wirtschaftlichen Freiheiten und sozialen Grundrechten im Lichte des sozialen Fortschritts, inoffizielle Übersetzung, 2008.

31 Zusammenfassend siehe Regina Viotto/Andreas Fisahn (Hrsg.): Europa am Scheideweg. Kritik des EU-,,Reformvertrages", in Kooperation mit dem wissenschaftlichen Beirat von Attac, Hamburg 2008. 
und von ihren Verpflichtungen Großbritannien und Polen ausnimmt) und die Einführung einer ,horizontalen Sozialklausel' (Art. 9 AEUV), durch die sich die Europäische Union verpflichtet, bei der Festlegung und Durchführung von Unionsmaßnahmen der Förderung von Beschäftigung und der Gewährleistung eines angemessenen Sozialschutzes, der Bekämpfung der sozialen Ausgrenzung sowie einem hohen Bildungsniveau und dem Gesundheitsschutz Rechnung zu tragen.

Andererseits schreibt der Reformvertrag im Bereich der Kompetenzen und Verfahren, also in den institutionellen Kernfragen, den Status quo ante weitgehend fort. Gerade die vertragliche Ermächtigungsgrundlage und die Frage von Einstimmigkeit oder Mehrheitsentscheidungen sind aber, wie die eingangs unternommene Kurzanalyse des sozialpolitischen Regierens zu zeigen versucht hat, von entscheidender Bedeutung, wenn es um das materielle Sekundärrecht und die regulatorischen Qualitäten der EU-Politik geht.

Dementsprechend gehen Prognosen über künftig mögliche sozialpolitische ,policy-outputs ' auch davon aus, dass diese aufgrund des gestärkten sozialen Normenkanons in erheblichem Maße durch ,Richterrecht' geprägt sein werden ${ }^{32}$ und weniger durch einen Zuwachs an substanziellen, nach der Gemeinschaftsmethode verabschiedeten neuen Richtlinien oder Verordnungen.

Versucht man dieses Gesamtergebnis in einen integrationspolitischen Langzeittrend der EU-Sozialpolitikentwicklung einzuordnen, so ist zunächst die folgende Besonderheit hervorzuheben:

In keinem anderen Politikfeld operiert (und experimentiert) die Europäische Union mit einem vergleichbar umfassenden Politikinstrumentarium wie im Bereich der, sozialen Dimension' der Europäischen Union. So umfassen die Modi der Steuerung und Regulierung, die in den Bereichen der europäischen Sozial-, Arbeits- und Beschäftigungspolitik zur Anwendung kommen, die ,klassische "EU-Gesetzgebung nach der Gemeinschaftsmethode vermittels Richtlinien und Verordnungen; distributive Politikansätze vermittels der Strukturfonds (arbeitspolitisch bedeutsam insbesondere der Europäische Sozialfonds; partiell der Globalisierungsfonds); die Selbstregulierung der europäischen Sozialpartner im Rahmen des Sozialen Dialogs; verschiedenste Maßnahmen, die - im weitesten Wortsinne - im Bereich des ,soft law' liegen (Empfehlungen, unverbindliche Rechtsakte etc.); und schließlich, als ,jüngster' sozialpolitischer Problemlösungsansatz die OMK.

Diese Vielfalt der Problemlösungsverfahren und der vielfach experimentelle und entwicklungsoffene Charakter einzelner Steuerungsinstrumente - dies gilt etwa für den Sozialen Dialog, insbesondere aber für die OMK - sind nicht etwa Ausdruck einer insgesamt ,starken ' EU-Governance im Bereich der europäischen Arbeits- und Sozialpolitik, sondern vielmehr Ausdruck tiefgreifender ordnungs- und integrationspolitischer Konflikte, politischer Blockaden und schwieriger Kompromissbildungsprozesse, die den Integrationsprozess gerade im Bereich der Ausgestaltung der sozialen Dimension von Beginn an begleiten. Wenn seit der Jahrtausendwende - auch mit Blick auf die sogenannte Lissabon-Strategie ,offene Steuerungsformen' in Gestalt der OMK extensiviert und intensiviert wurden, so reflektiert dies eine Situation, in der die mit der Währungsunion gewachsene sozial-ökonomische Interdependenz zwischen den Mitgliedstaaten einen objektiv erhöhten Koordinierungsund Steuerungsbedarf erzeugt (,management of interdependence'), während gleichzeitig, im Zuge der Osterweiterung, das ,management of diversity “ einen strukturell bedingten Komplexitätsgrad angenommen hat, der eine verbindliche sozialpolitische Regulierung und hier-

32 Treib: Der EU-Verfassungsvertrag, 2004. 
archische Steuerung im bisherigen Vertragsrahmen von Nizza zunehmend erschwert oder gar unmöglich macht.

Hier nun kommt die konstitutionelle Dimension ins Spiel, nämlich die Frage einer Anpassung des EU-Kompetenz- und Entscheidungsgefüges an die Bedingungen einer ökonomisch-monetär vertieften Integration und zugleich an eine in ihrer Mitgliedschaft erweiterten und sozial-ökonomisch heterogeneren Europäischen Union. Geht man von einer ,eurooptimistischen" wissenschaftlichen Position aus, wonach die Europäische Union schon auf der Grundlage des Nizza-Vertrages ,[...] über ausreichende Kompetenzen (verfügt), um eine aktive Sozialpolitik zu praktizieren. Die Kompetenzen werden lediglich nicht optimal genutzt " ${ }^{33}$ so wird man einen weitergehenden konstitutionellen Reformbedarf eher gering veranschlagen. Hält man hingegen am Projekt einer politischen Union fest, zu deren integralem Teil erweiterte und gestärkte wirtschafts- und sozialpolitische EU-Steuerungskompetenzen gehören müssten, so ist die Europäische Union - geht man vom Maastricht-Vertrag aus - innerhalb der ,ersten Säule“ so gut wie nicht vorangekommen (dies im Übrigen im Gegensatz zu integrationsvertiefenden Schritten, die im Bereich der ,zweiten“ und ,dritten Säule" seit Maastricht möglich waren). Nimmt man schließlich empirisch gewichtige wissenschaftliche Diagnosen hinzu, wonach die politische Ökonomie der gegenwärtigen Europäischen Union die Mitgliedstaaten in die Rolle von ,Wettbewerbsstaaten " mit problematischen wohlfahrtsstaatlichen Folgen getrieben hat, ${ }^{34}$ so erscheinen konstitutionelle Reformschritte, die das asymmetrische Verhältnis zwischen den ,marktschaffenden“ und ,marktkorrigierenden “ Unionskompetenzen ausgleichen, zwingend geboten. Neben dem konstitutionellen Minimalismus im Bereich der EU-Sozialpolitik (siehe dritter Teil AEUV, Titel IX bis XI) weist der Post-Nizza-Prozess jedoch auch in , angrenzenden' Politikfeldern (Binnenmarkt, Steuerpolitik, Koordinierungs- und Steuerungsregeln in makro-ökonomischen Bereichen etc.) einen weitgehend unveränderten vertraglichen Kompetenzrahmen auf (siehe dritter Teil AEUV, Titel I bis VIII).

Im Gegensatz zu einzelnen demokratiepolitischen Fortschritten (,doppelte Mehrheit", Einbeziehung der nationalen Parlamente etc.) und Erfolgen, die mit der Verankerung neuer zukunftsrelevanter Kompetenzfelder (Energie, innere Sicherheit etc.) erreicht werden konnten, ${ }^{35}$ lassen die Prozessverläufe und Ergebnisse in den sozial-ökonomischen Politikfeldern deutliche Stagnationstendenzen erkennen. Hier befindet sich die EU-27 offensichtlich im Stadium eines Equilibriums, allerdings im Sinne eines , ungleichgewichtigen Gleichgewichts'.

Dieses vertragspolitische Stadium bekräftigt die eingangs formulierte Annahme einer sozialpolitischen Kompetenztransferfalle, in der sich die Europäische Union aufgrund folgender Beobachtungen befindet: Trotz der Erwartung, die Währungsunion würde politische Spill-over-Effekte in Richtung eines supranationalen sozial-ökonomischen Steuerungsbedarfs erzeugen und trotz entsprechender Forderungen einzelner Mitgliedstaaten und verschiedener gesellschaftlicher Kräfte, die Binnenmarkt- und Währungsintegration sozialpolitisch und makro-ökonomisch stärker zu flankieren beziehungsweise ,einzubetten', war es in

33 Regine Prunzel: Der „Europäische Sozialkonsens” als Instrument zur Stärkung des „Europäischen Sozialmodells“. Vom politischen Postulat zur eigenständigen sozialpolitischen Säule, Berlin 2007, S. 409.

34 Siehe unter anderen Klaus Busch: „Das Korridormodell - ein Konzept zur Weiterentwicklung der EU-Sozialpolitik“, in: Josef Schmid/Reiner Niketta (Hrsg.): Wohlfahrtsstaat. Krise und Reform im Vergleich, Marburg 1998, S. 273-295; Patrick Ziltener: Strukturwandel der europäischen Integration. Die Europäische Union und die Veränderung von Staatlichkeit, Münster 1999; Fritz W. Scharpf: The European Social Model: Coping with the Challenges of Diversity, in: Journal of Common Market Studies 4/2002, S. 645-670.

35 Zum gesamten Vertragswerk siehe Werner Weidenfeld (Hrsg.): Lissabon in der Analyse. Der Reformvertrag der Europäischen Union, Baden-Baden 2008. 
den jüngeren Vertragsverhandlungen (im Grunde genommen seit Maastricht ${ }^{36}$ ) nicht möglich, Mehrheiten zu finden, die signifikante Veränderungen in der wirtschafts- und sozialpolitischen Kompetenzorganisation der Europäischen Union ermöglicht hätten.

Es sind verschiedene Faktoren, die diese Kompetenztransferfalle erklären beziehungsweise das konstitutionelle sozialpolitische Dilemma zwischen dem Subsidiaritätsgebot und nationalen Souveränitätsvorbehalten einerseits und dem Kohäsions- und Solidaritätsanspruch der Europäischen Union andererseits begründen. Wie an einzelnen Stellen dieses Beitrags bereits gezeigt, zählt dazu die Affinität der nationalen Sozialbürger zu ,ihren` (institutionell und normativ) zudem höchst unterschiedlich ausgeprägten Wohlfahrtssystemen. Hinzu kommen ,ein unionsweit differentielles und insgesamt nur mäßig ausgeprägtes Gefühl, einander solidarisch verpflichtet und verbunden zu sein, [...] sowie schließlich , Ressentiment und Indifferenz' gegenüber der EU-Erweiterung “. ${ }^{37}$

Auf der Ebene der Regierungen spiegelt sich die strukturelle Diversität der nationalen Wohlfahrts- und Arbeitsrechtsregime in entsprechenden Kalkülen bezüglich der Anpassungskosten, die mit einer Ausweitung sozialpolitischer EU-Regelungen verbunden wären. Entsprechend defensiv verhalten sich mittlerweile nicht nur die ,traditionellen Bremser ${ }^{\text {* }}$ Großbritannien und Irland sondern auch einzelne der neuen mittel- und osteuropäischen Mitgliedstaaten (dezidiert Tschechien und Estland). Beim Konsens- und Einstimmigkeitszwang, der bei Vertragsveränderungen gegeben ist, sind hierdurch politische Blockaden programmiert, zumal dann, wenn der Kreis der Vetospieler, wie dies im Zuge der EU-Osterweiterung der Fall war, größer wird.

Unter diesen vertragspolitischen Bedingungen zeichnen sich bei der künftigen gemeinschaftlichen Bewältigung sozialpolitischer und makro-ökonomischer Herausforderungen, die nach aller Voraussicht durch die Folgen der Weltfinanzmarktkrise wachsen werden, unterschiedliche Entwicklungsszenarien ab.

Denkbar ist zum einen, dass die nach dem Reformvertrag erleichterten Optionen , abgestufter Integration“, die die „Flexibilitätsklausel“ (EUV Titel IV) bietet, stärker als bisher in Anspruch genommen werden. Etwa dadurch, dass sich eine Staatengruppe mit sozialstrukturell ähnlichen Voraussetzungen und gemeinsamen transnationalen Problemlösungsbedarfen zu entsprechenden Vertiefungsschritten einer sozialpolitischen Regulierung zusammenfindet oder auch dadurch, dass sich die Mitglieder der Eurozone auf erweiterte finanz- und wirtschaftspolitische Steuerungsformen verständigen, die zugleich wohlfahrtspolitischen Zielen dienen könnten.

Zum anderen werden gemeinschaftliche Politiken im Rahmen der EU-27 nach aller Voraussicht in noch stärkerem Maße auf jenen Instrumenten und Verfahren des ,governancelearning', der ,soft regulation " und der Rahmenkoordination basieren, die, wie eingangs analysiert, sich schon seit der Jahrtausendwende in Gestalt der OMK herausgebildet haben und die mittlerweile in einer wachsenden Zahl von Politikfeldern zur Anwendung kommen. Der Reformvertrag präzisiert und fixiert - ohne den Begriff OMK zu verwenden - die diesbezüglichen Verfahren im Vertragstext (Art. 156 AEUV).

$\mathrm{Zu}$ den Vorteilen der Steuerungsform der OMK zählt, dass sie auch die Einbeziehung von Politikfeldern und -materien ermöglicht, in denen die Europäischen Union keine originären Rechtssetzungskompetenzen besitzt, die aber gleichwohl in einem Interdependenzzusammenhang mit gemeinschaftlichen Zielen und Projekten der Europäischen Union stehen, wie sie etwa in der Lissabon-Strategie formuliert sind.

36 Platzer: Deutschland und die europäische Sozial- und Beschäftigungspolitik, 2001.

37 Prunzel: Der „Europäische Sozialkonsens”, 2007, S. 362. 
Ob die OMK tatsächlich „ein Verfahren zur Verbindung von wirtschaftlicher Effizienz mit sozialer Gerechtigkeit sowie eine Autonomie schonende Alternative zu supranationaler Regulierung und intergouvernementaler Entscheidungsfindung [... $]^{\text {33 }}$ ist beziehungsweise ob und wie sie dahingehend (weiter)entwickelt werden kann, ist wissenschaftlich und politisch strittig.

So wird beispielsweise in der jüngeren politikwissenschaftlichen Governance-Debatte ,diskursiven Regulierungsmechanismen' und nicht-hierarchischen Steuerungsformen eine große Bedeutung für , gutes ‘ und ,modernes ' Regieren zugemessen. Zumal mit Blick auf die besonderen Systemeigenschaften der Europäischen Union, in der die deliberative Politik eine beträchtliche Rolle spielt, ${ }^{39}$ sehen einzelne Autoren in der OMK ein geradezu ideales, EU-konformes Steuerungsinstrument, das eine ,experimental governance ' ermögliche, konsensuale sozialpolitische Problemlösungsstrategien generiere und einen , pragmatic constitutialism' befördere. ${ }^{40}$

Die hier unternommene Analyse legt ein skeptischeres Zukunftsszenario nahe: Im Kontext der sozialpolitischen Kompetenztransferfalle erscheinen die verschiedenen OMK-Verfahren zwar umso unverzichtbarer, als sie eine Art ,Notausgang' oder ,Umgehungsstrecke ‘ darstellen (können). Gleichwohl lassen die Ziele der OMK, die primär in einem ,policyoder ,governance-learning ' bestehen, und die - letztlich voluntaristische - Verfahrenslogik der OMK eine ,pragmatische Konstitutionalisierung ' vor allem dann nicht erwarten, wenn es sich um zwischenstaatlich sensible - und das heißt, mit (Anpassungs-)Kosten verbundene - Politikfelder handelt, die sich schon zuvor bei Vertragsreformen als ,nicht verhandelbar erwiesen haben und vertragspolitisch einer supranationalen Steuerung oder Vergemeinschaftung entzogen wurden.

38 Ingo Linsenmann/Christoph Meyer: Dritter Weg, Übergang oder Teststrecke? Theoretische Konzeption und Praxis der offenen Politikkoordinierung, in: integration 4/2002, S. 285-296, hier S. 285.

39 Burkhard Eberlein/Dieter Kerwer: Theorising the new Modes of European Union Governance, in: European Integration online Papers 6/2002.

40 Charles Sabel/Jonathan Zeitlin: Active Welfare, Experimental Governance, Pragmatic Constitutionalism: The new Transformation of Europe, Konferenzpapier für die Konferenz ,The Modernisation of the European Social Model and EU Policies and Instruments“, Ionnanina 2003. 